University of Massachusetts Amherst

ScholarWorks@UMass Amherst

Publications

MURI on Photomechanical Materials

2019

Light-induced shape morphing of thin films

Alexa S. Kuenstler

University of Massachusetts Amherst

Ryan C. Hayward

University of Massachusetts Amherst, hayward@umass.edu

Follow this and additional works at: https://scholarworks.umass.edu/muri_pubs

Kuenstler, Alexa S. and Hayward, Ryan C., "Light-induced shape morphing of thin films" (2019). Current Opinion in Colloid \& Interface Science. 3.

https://doi.org/10.1016/j.cocis.2019.01.009

This Article is brought to you for free and open access by the MURI on Photomechanical Materials at ScholarWorks@UMass Amherst. It has been accepted for inclusion in Publications by an authorized administrator of ScholarWorks@UMass Amherst. For more information, please contact scholarworks@library.umass.edu. 


\section{Light-Induced Shape Morphing of Thin Films}

Alexa S. Kuenstlera and Ryan C. Hayward ${ }^{\star, a}$

aDepartment of Polymer Science and Engineering, University of Massachusetts

Amherst, Amherst, Massachusetts 01003, United States

*E-mail: hayward@umass.edu

\section{Abstract}

Shape transformation of thin two-dimensional sheets into three-dimensional structures using light is of great interest for remotely-controlled fabrication, surface modulation, and actuation. Over the last few decades, significant efforts have been made to develop materials systems incorporating photochemical or photothermal elements to drive deformation in response to illumination. However, the full extent of the interplay between chemistry, optics, and mechanics in these materials is poorly understood. In this Review, we introduce principles of shape morphing in these systems by considering the underlying physics of photo-induced stresses and how these have been used in recent literature. In addition, we provide a critical overview of the important design characteristics of both photochemical and photothermal system and offer our view on the open opportunities and challenges in this rapidly growing field.

\section{Keywords}

Photomechanics, polymers, soft materials, actuators, shape morphing, thin film mechanics, light responsive materials 


\section{Introduction}

The out-of-plane buckling of thin elastic sheets in response to non-uniform stresses is a powerful method to control both the form and function of synthetic materials. While such 'morphogenesis' of two-dimensional membranes into three-dimensional shapes can be driven by many cues including mechanical, chemical, and electrical stimuli [1], light represents a particular powerful means of control thanks to the ability to deliver photons wirelessly and rapidly over long-distances. Just as photons from the sun travel millions of miles to power life on Earth, photons from incoherent or coherent sources can be similarly delivered through free-space or optical fibers to drive the response of a photoactive material. Additionally, spatio-temporal control over the wavelength, mode, and polarization of the light provides a number of independent degrees-of-freedom that can be exploited to define complex and dynamic optomechanical responses. However, the efficient and practical transformation of light energy into prescribed shape changes remains challenging.

Two main approaches have typically been exploited to generate deformation in photoactive materials: photochemical transformations and photothermal heat generation. While many examples of the synthesis and actuation of photomechanical materials exist in the literature, there remain large gaps in our understanding of how to rationally engineer these materials to achieve a desired photoresponse. The aim of this review is to demonstrate the state-of-the-art in photomechanical materials through specifically selected examples and to critically examine the distinct physics that govern the response 
of photochemical versus photothermal materials. We do not intend this review to be a historical nor comprehensive overview of the field; for this, we direct the reader to a number of recent publications that cover several decades of work [2-5]. We begin by presenting general strategies for shape control by engineering strain fields and leveraging optical properties in thin sheets (2). We follow with an overview of canonical examples and recent advances in photomechanical materials, as well as a critical analysis of the underlying physics in photochemical versus photothermal systems (3-5). Finally, we close by offering our perspective on the important design characteristics and future avenues for development in this exciting class of materials (6).

\section{Basics of Shape Programming with Light}

In this section, we present an overview of how elastic energy, geometry, and optics couple to generate dynamic shape change in thin sheets.

\subsection{Patterned Bending and Mean Curvature}

A thin elastic sheet subjected to stress will tend to buckle out of plane to lower its elastic energy, thanks to the stronger scaling of bending $\left(E_{B} \sim t^{3}\right)$ compared to stretching $\left(E_{s} \sim t\right)$ energy with thickness $t$. Bending provides a route to alter the mean curvature of the sheet, defined as $H=\left(k_{1}+k_{2}\right) / 2$, where $k_{1}$ and $k_{2}$ are the principle (maximum and minimum) curvatures at any given point. As illustrated in Fig. 1 this can be achieved by non-uniform strains through the thickness of the film, as described by Stoney [6] and Timoshenko for bilayers [7]. This variation in strain can be pre-programmed into a thin film in any number 
of ways including through-thickness variations in the thermal expansion, degree of swelling, or ordering/orientation of a liquid crystalline moiety, either as a smooth gradient or due to a contrast between discrete layers. However, in photomechanical materials, the decay of light intensity I through the thickness of a film provides a natural method to generate non-uniform strain, even for an otherwise homogeneous material. Specifically, for an absorbing layer one expects that $I(y)=l_{0} \exp \left(-\mu_{t} y\right)$, where $l_{0}$ is the incident intensity, $y$ is the spatial coordinate along the direction of light propagation, and $\mu_{\mathrm{t}}$ is the attenuation coefficient. For a film of thickness $t>\mu_{\mathrm{t}}^{-1}$, most photons are initially absorbed at the surface of the film, generating a gradient in deformation and therefore a spontaneous curvature.

An important consideration in programmed bending is that of "incompatible curvatures". In the case of materials with in-plane isotropy, such as many hydrogels, the preferred curvature defined by a through-thickness variation is the same along every direction. However, satisfying this preference to curve isotropically would require a change in Gaussian curvature into a spherical shape, and therefore significant stretching (as described in more detail below). Thus, under most conditions the bilayer will instead bend along only one direction, as selected by its in-plane geometry. For example, a rectangular sheet will generally have an equilibrium configuration with curvature along its longer direction, due to edge effects (Figure 1B) [8], although kinetic factors can often lead to mechanically stable states that bend along other directions. The introduction of anisotropy into the material, as in liquid crystalline polymers or many photomechanical crystals, 
1. Mean Curvature

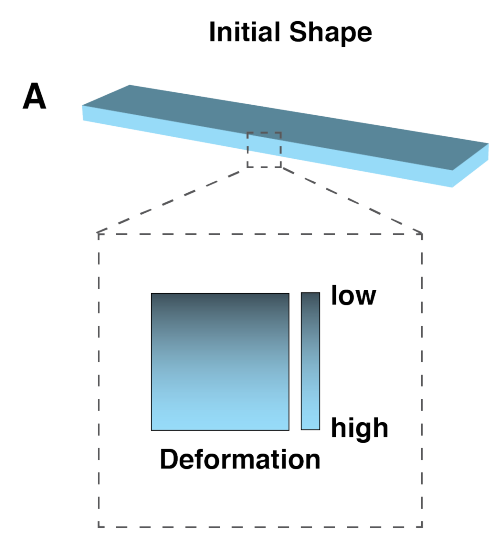

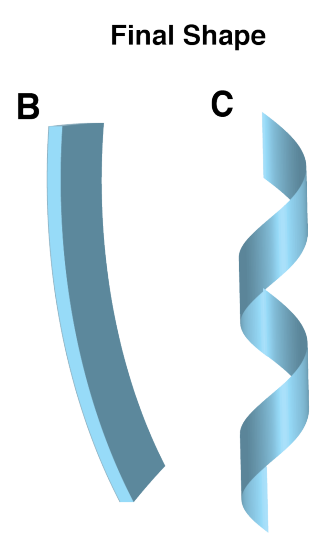

2. Gaussian Curvature

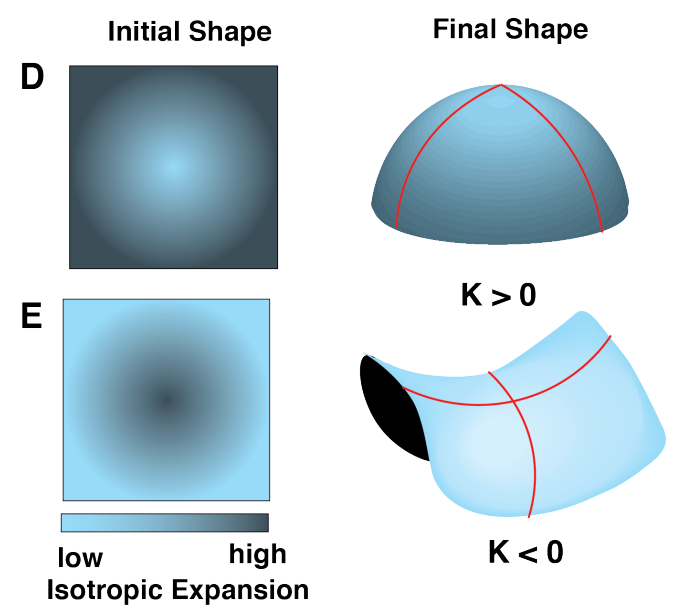

Figure 1. Overview of mean and Gaussian curvature. (A) In a photomechanical strip, mean curvature is generated via non-uniform expansion through the thickness of the strip. Deformation gradients can be pre-programmed through variations in material properties (i.e. swelling, thermal expansion, or molecular ordering) or it can arise spontaneously as a result of light absorption through the film thickness. (B) To avoid stretching, an isotropic material will bend along a single direction, usually specified by its in-plane geometry. (C) Introduction of anisotropy specifies bending along the axis with the largest magnitude of preferred curvature, often generating helical shapes. (D) Patterning of Gaussian curvature in thin films can be achieved through in-plane variations in deformation. In the case of high expansion in the middle and low expansion at the edges, the film will buckle into an elliptical shape with positive Gaussian curvature. (C) In the opposite case, the film with buckle into a hyperbolic (saddle-like) shape with negative Gaussian curvature.

inherently breaks this symmetry, such that the ground state generally corresponds to bending along the principal direction with the larger magnitude preferred curvature. For example, a thin ribbon with principle curvatures oriented at $\pm 45^{\circ}$ relative to its long axis will generally adopt a helical shape as shown in Figure $1 \mathrm{C}$.

\subsection{Patterned Stretching and Gaussian Curvature}

Achieving more complex shapes that simultaneously curve along both directions requires programming of the Gaussian curvature $K=k_{1} k_{2}$, which geometrically requires that there be non-uniform in-plane stretching, compression, or shear [9-11]. For example, in an isotropic material such as a hydrogel, an appropriate gradient from large swelling in the 
middle of a sheet to low swelling at the edges (controlled, e.g., by patterning the crosslink density or hydrophilicity of the gel) can yield a spherical cap with $K>0$ (Figure 1D), whereas the opposite configuration generates a saddle with $K<0$. Similar principles hold for anisotropic materials such as liquid crystal elastomers (LCEs), although in this case the orientation of the material can also be varied along the in-plane directions to pattern shape. For example, a circular disk that contracts more along the azimuthal direction than the radial direction (e.g., an LCE with the director oriented azimuthally around a +1 defect) will buckle into a conical shape that possesses a $K>0$ singularity at its tip, while the opposite configuration will yield a saddle-like 'excess'-cone with a $K<0$ singularity at the origin.

Light-induced patterning of Gaussian curvature can generally be approached in one of two basic ways: (1) uniform illumination of a film with a "blue-printed" 3D shape defined by pre-programmed in-plane variations in deformation, or (2) non-uniform illumination of a homogeneous film using spatially-patterned light. Although the latter approach offers the possibility to continuously reprogram a single sheet of material into an arbitrarily large number of 3D shapes, the design rules for programming shapes in this manner remain far less established. In addition, the possibilities offered by combining elements of these two approaches remain largely unexplored.

Notably, specifying $K$ alone is insufficient to fully dictate three-dimensional shape. For example, a spherical cap can either buckle upwards with $H>0$ or downwards with $H<0$ 
but have the same Gaussian curvature in either case; for more complex geometries, this can yield a multitude of mechanically stable configurations with qualitatively different shapes. Thus, fully general strategies for shape programming require routes to simultaneously prescribe both mean and Gaussian curvature, a challenge that has so far been met in only a very few cases for thermally actuated materials [10-13], and to our knowledge has not yet been addressed in light-responsive systems.

\section{Photochemical Materials}

Photochemical materials undergo a chemical transformation in response to light, often leading to a change in geometry and/or connectivity at the molecular level, as shown in Figure 2 for several major classes of materials. If these molecules can be organized to rearrange in a cooperative fashion, these Angstrom-level chemical changes can be harnessed to drive macroscopic deformation. Typical strategies include incorporating photochemical molecules

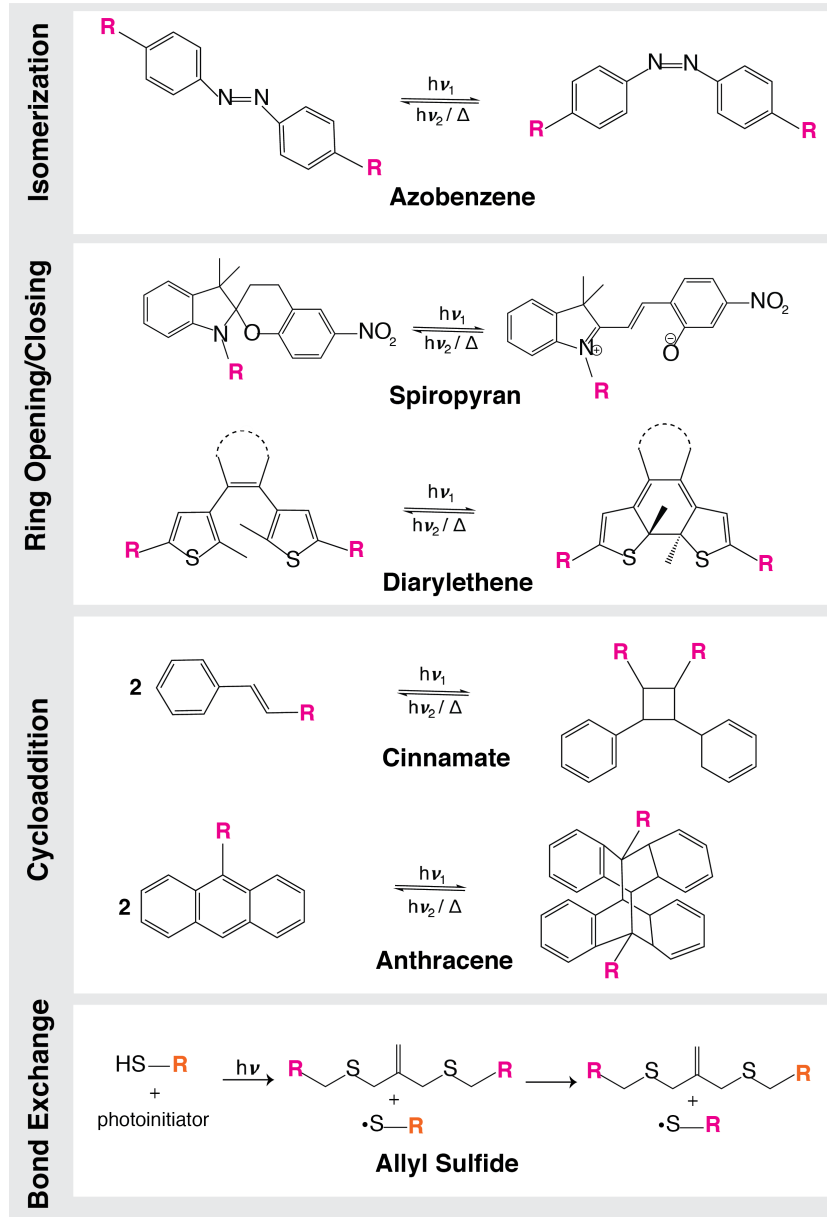

Figure 2. Examples of photochemical elements 


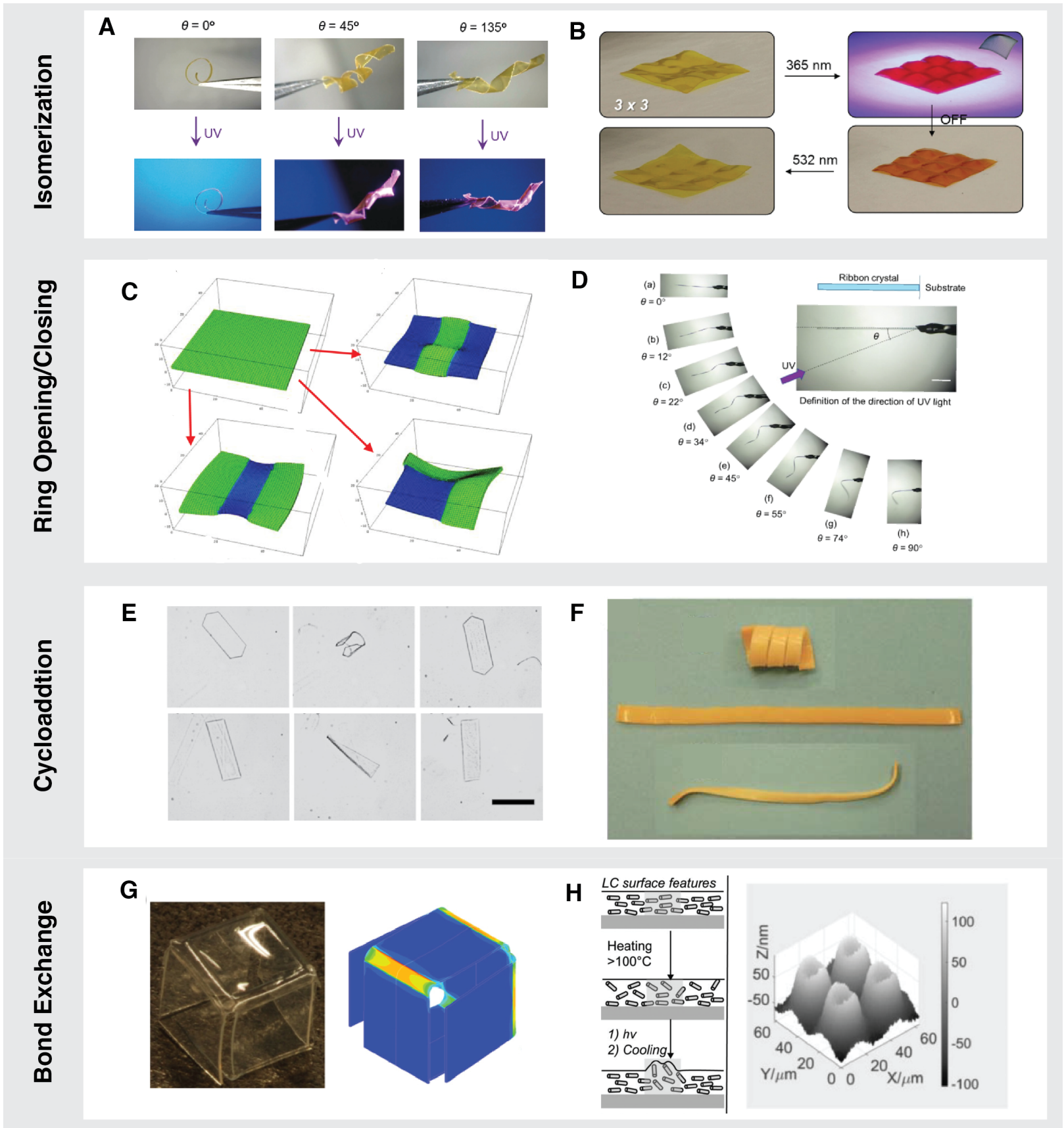

Figure 3. Examples of photochemically-driven shape change. (A) Actuation of nematic LCN ribbons (reproduced from ref.[18]; copyright 2017 Wiley). (B) Reversible light-driven shape morphing of an array of +1 defects in an azobenzene-containing LCE (reproduced from ref. [19]; copyright 2016 Wiley). (C) Simulation of photopatterned actuation of spiropyran-containing gels (reproduced from ref. [25]; copyright 2013 Wiley). (D) Photo-directed curling of a diarylethene ribbon (reproduced from ref. [30]; copyright 2018 American Chemical Society). (E) Bending and twisting of anthracene nanoplatelets with different molecular orientations (reproduced from ref. [33]; copyright 2018 Wiley). (F) Dimerization-induced curling (top) and relaxation (bottom) of a cinnamate-containing amorphous polymer (reproduced from ref. [35]; copyright 2005 Springer Nature). (G) Photo-induced folding of a pre-strained thiol-ene polymer (reproduced from ref. [37]; copyright 2012 American Institute of Physics. $(\mathrm{H})$ Surface feature development in an allyl sulfide-containing LCN (reproduced from ref. [38]; copyright 2017 Wiley). 
into matrices capable of undergoing thermodynamic phase transitions involving changes in size or shape, growing single crystals of photomechanical molecules, or exploiting changes in covalent bonding to generate or dissipate stress. In this section, we review photochemical systems based on four photochemical mechanisms: 1) cis-trans isomerization, 2) ring opening and closing, 3) cycloadditions, and 4) bond exchange.

\subsection{Cis-trans Isomerization}

Cis-trans isomerization corresponds to the transformation of a molecular configuration by rotation about a double bond. Azobenzene is among the most widely studied photochromes of this class and can switch reversibly from a thermally-stable extended trans-state to a bent cis-state upon absorption of a photon (Figure 2). The reverse cis to trans isomerization can happen either by thermal relaxation or absorption of a photon at another wavelength. The photophysics of these processes can be tailored through ortho and para substitutions of the phenyl rings, as will be addressed in Sections 5 and 6 . While early work on azobenzene-containing materials primarily made use of amorphous matrices doped with photoactive units, the use of ordered matrices, in particular liquid crystalline networks (LCNs), provides a more powerful means to drive large shape changes. Upon conversion from the trans to cis forms, the change in azobenzene shape tends to disrupt the local ordering of the surrounding nematic network, resulting in a contraction along the director. Importantly, light absorption and repeated photoisomerization also inevitably generate heat (as will be addressed in detail in Section 4.1), and while photomechanical effects in many azobenzene-functionalized LC materials are 
largely attributed to isomerization, it can often be difficult to fully deconvolute the photochemical and photothermal contributions to the observed photoresponse [14].

Pioneering efforts by Finkelmann's group established the amplification of photomechanical shape change that could be achieved via isomerization-induced disordering using azobenzene-containing side-chain liquid crystal elastomer with a siloxane backbone, yielding a $\sim 20 \%$ linear contraction along the director upon exposure to UV light [15]. Ikeda's group realized reversible 3D shape changes in polydomain LCNazobenzene films [16] through selective absorption of polarized light at the film surface by azobenzene units aligned along the polarization direction, leading to a slight contraction along this direction at the top surface, and therefore bending along a defined axis. Beyond the linear actuation and bimorph-like bending demonstrated in this pioneering work, prescription of director orientation can be exploited to control helical twisting or formation of shapes with non-zero Gaussian curvature. Following their seminal work in the photoactuation of twisted nematic LCN ribbons into helices of controlled pitch and handedness using a chiral dopant [17], Katsonis and co-workers recently introduced a particularly elegant method for fabricating bio-inspired photoresponsive chiral helices from achiral substituents [18]. In this work, azobenzene-containing LCNs were polymerized with alternating stripes of ordered and unordered regions with a throughthickness gradient in crosslinking density introduced during photopolymerization. Isotropic and anisotropic polymerization-induced shrinkage in the disordered and ordered regions, respectively, gives rise to incompatible curvatures that are satisfied by buckling 
into helices. As shown in Figure $3 \mathrm{~A}$, when the stripes were offset by $45^{\circ}$ from the long axis of a ribbon, the material twisted into a helix to satisfy one of the curvatures, with the direction of buckling and handedness dictated by the through-thickness gradient. When exposed to UV light, azobenzene isomerization caused the ordered stripes to contract significantly along the director but the disordered stripes to deform isotropically, and the helices coiled more tightly. Finally, inspired by seed pod mechanics, helices of opposite handedness were coupled to form a tube that spontaneously burst open due to the buildup of photo-induced strain.

To achieve dynamic optical control of shapes with $K \neq 0$, complex in-plane director orientation in photo-responsive LCEs can be coupled with spatiotemporally-patterned light. Recently, White et al. extended their approach to dictate arbitrary director fields with photo-patterned alignment layers to photo-responsive azobenzene-containing LCE matrices [19]. Azobenzene-containing LCE samples were prepared with +1 azimuthal, +1 radial, and -1 defects. At steady-state, uniform UV light absorption through the film thickness drove out-of-plane buckling into cone-like, saddle-like, and hemisphere-like shapes due to contraction along the director. As shown in Figure 3B, when multiple +1 defects were patterned in the same sheet, flood illumination of the entire film drove the formation of an array of conical domes, due to the more extensive contraction in the azimuthal direction around each defect, as described in Section 2. Finally, local illumination of the region surrounding just one of the defects was shown to yield selective activation of only that cone, providing dynamic reconfiguration into several different 
shapes.

In addition to incorporation in polymer matrices, efforts have also been made to assemble azobenzene derivatives into photomechanical crystals, although there is less work in this area. This approach has proven challenging because it is difficult to accommodate the large shape change necessitated by isomerization in tightly packed crystal lattices. One successful approach is to localize isomerization to the surface of the crystal, as shown by Uchimoto and co-workers in micron-scale plate-like crystals of 4(dimethylamino)azobenzene, giving rise to reversible bending [20]. Other examples of photomechanical azobenzene crystals include perhalogenated systems [21] and 'pushpull' pseudostilbenes [22]. Finally, a particularly promising involves the use of cocrystallization to engineer the crystal properties. For example, Bushuyev et al. have shown that azobenzenes functionalized with halogen bond donors and pyridine-based acceptors form co-crystals with a wide range of photomechanical behaviors [23]. Thus, while incorporation of azobenzene into polymer matrices remains the most common method to generate photomechanical responses from these compounds, photomorphing of azo-based molecular crystals is also possible and worthy of further study.

\subsection{Ring Opening and Closing}

Unlike cis-trans isomerization, where the molecular conformation is distorted but the bond connectivity is unchanged, photochemical changes can also occur through the formation and breaking of covalent bonds. The first class of these reactions we will consider are intramolecular ring opening and closing reactions. While many examples of these 
molecules exist in the literature, we will only consider two particularly widely used molecules of this class: spiropyran and diarylethene.

Spiropyran reversibly ring-opens upon exposure to UV light to a highly polar merocyanine form. This change in polarity is often exploited in hydrogel systems to drive photoresponsive swelling [24]. When incorporated into poly( $N$-isopropylacrylamide) (PNIPAAm) gels and placed in acidic aqueous solutions, spiropyran is primarily in the hydrophilic protonated open-ring form. Upon exposure to visible light, the ring closes and becomes hydrophobic, driving deswelling. While early work demonstrated the utility of this platform to drive homogeneous volumetric swelling changes, pioneering simulations by Balazs and co-workers demonstrated the possibilities for complex, reconfigurable shape changes of thin spiropyran-doped gels under spatiotemporal light patterns [25]. As shown in Figure 3C, a single stripe down the center of the sheet was shown to generate in-plane variations in swelling between illuminated and non-illuminated regions, driving reversible buckling into hourglass-like shapes that could be tailored by the size and location of the stripe as the gel seeks to relieve interfacial strain between swelled and deswelled regions. Similarly, square patterns of light drove more complex twisting and curvature, as demonstrated by the saddle-like shape adopted by a gel with one corner illuminated. Remarkably, the authors also predicted that directed motion could be achieved using spatiotemporally-controlled light patterns. For example, periodically rastering a stripe of light along the gel in a consistent direction was found to yield translation of the gel sheet as a result of peristalsis-like deformation. (While this approach 
has not to our knowledge been experimentally demonstrated in spiropyran-containing gels, work in our group on photothermally responsive gels [26] has shown similar behaviors, as described in the next section.) Additional experimental work by Schenning and co-workers demonstrated the utility of spiropyran-containing gels to drive non-trivial and on-demand shape change [27]. By exploiting polymerization-induced diffusion, they prepared light-responsive gels with spatially-patterned crosslink densities. By polymerizing the gels directly on pre-structured substrates such that the gels were constrained, they made block-like, wave-like, and ratchet-like topographies upon photodriven deswelling. Furthermore, the magnitude of the protrusions could be controlled by light intensity. This work demonstrates that in addition to exploiting geometrical changes of molecular photoswitches to drive shape change, similar effects can also be achieved through the use of photoresponsive additives that dynamically switch their chemical properties.

Another commonly used class of molecules in ring opening and closing reactions are diarylethene derivatives, which contain three heterocyclic rings that switch between ring open and closed isomers upon exposure to distinct wavelengths of light. Significantly, both isomers are thermally stable, making these molecules particularly well-suited to shape fixing. While diarylethenes have been employed in LCNs to drive photoactuation [28], they are more commonly assembled into photomechanical crystals that undergo shape changes in response to photochemical reactions. Importantly, assembly into crystals results in precise, long-range ordering of a high density of photoactive molecules 
that can be harnessed to produce highly concerted responses difficult to achieve in polymer matrices with dilute concentrations of chromophores. The first demonstration of photoactive diarylethene crystals was by Irie and coworkers, who demonstrated that upon ring-closing in diarylethene derivatives they could reversibly transform $100 \mu \mathrm{m}$ square single crystals into a rhombus shape or bend micron-sized rods depending on molecular substituents [29]. The crystal deformation is driven by changes in molecular packing due to planarization of the diarylethene upon ring-closing, driving contraction along one crystallographic axis and expansion along another. In the diarylethene rods, a gradient of photoisomerization through the thickness of the rod drives generates inhomogeneous contraction that drives bending in accordance with bilayer mechanics. Since then, rich mechanical behavior has been demonstrated in these systems and the observed mode of deformation can be tailored by crystal geometry and molecular substituents. Recently, Kobatake's group demonstrated that the dynamic response of these materials can be tuned by the direction of illumination in a similar fashion to azobenzene-LCN systems [30]. They prepared ribbon-like diarylethene crystals via sublimation that organized with alternating layers of molecules organized at $20^{\circ}$ and $-20^{\circ}$ with respect to the axis of the ribbon. When uniformly illuminated, these crystals twisted as a result of orthogonal contractions of the two oriented sub-populations, introducing similar mechanics to that of twisted nematic LCN ribbons. Remarkably, as the illumination angle with respect to the top of a diarylethene ribbon changed, the ribbon gradually transformed from a helicoid to a cylindrical helix shape and the handedness of the twist could also be controlled (Fig. 3D). Shape selection was attributed to selective activation of molecules whose dipole 
moments were aligned parallel to the direction of light propagation, thus generating different stress tensors and preferred curvatures with respect to the long axis of the ribbon. Significantly, while systems like twisted-nematic ribbons generate a single selected shape based on the prescription of director orientation, this system can dynamically switch between a helicoid or a cylindrical helix via selective activation of differently-oriented photoactive populations.

\subsection{Cycloaddition}

Similar to ring opening and closing, cycloaddition reactions drive changes in spatial conformations of molecules upon ring formation. However, unlike the intramolecular reactions considered above, cycloadditions involve the intermolecular reaction of unsaturated molecules to form cyclic adducts. Of particular interest are [2+2] and [4+4] reactions, where dimerization results in the formation of four- and eight-membered rings, respectively. In this section, we consider two canonical molecules employed in photomechanical systems: anthracene and cinnamate.

Anthracene derivatives form thermally-reversible dimers via a [4+4] cycloaddition upon UV light absorption. Similar to diarylethene, these molecules can be self-assembled into crystals that undergo shape change in response to photodimerization, the shape selection of which are highly sensitive to the crystal morphology, packing, and molecular substituents. While different anthracene derivatives vary in their kinetic responses, forwards/backwards efficiencies, and molecular packing, crystal morphology largely 
determines the macroscopic shape change of these crystals. As shown in Figure 3E, work by Bardeen and co-workers showed that microneedles bend while microribbons twist in derivatives of anthracene containing methyl or carboxylic acid groups, the extent of which is controlled by illumination conditions [31,32]. They studied the shape evolution of such microneedles and microribbons of 9-methylanthracene, and found that at intermediate illumination times, the needles bend and the ribbons twist due to stress build-up between distinct regions of photoreactants and products [31]. However, as illumination continues, more dimers are formed and the crystals gradually straighten out to their original shape as interfacial strain between photo-populations is relieved. To explain these observations, they developed a simple model to deduce the distribution of strain within the crystal as a function of dimer population and used this to trace the curvature evolution over time using bilayer bending mechanics. While the twisting behavior was not considered in detail, presumably it arises due to curvature induced by distinct populations of monomer and dimers, the orientation of which leads to a maximum contraction off-axis. Recently, Tong et al. have exploited curling of anthracene nanoplatelets upon UV exposure to achieve directed motion [33]. The nanoplatelets reached a maximum curvature at an intermediate point in the light-induced reaction when the internal strain mismatch is at a maximum. By ceasing illumination at this point, the authors used this shape change to trap aggregates of superparamagnetic nanoparticles. Subsequent application of a magnetic field caused the crystals with trapped aggregates to translate while uncurled microplatelets remained stationary. Further UV exposure caused the nanoplatelets to uncurl and released their cargo as the reaction went to completion and the internal strain due to distinct populations 
of monomers and dimers was relieved.

Cinnamates, which undergo a [2+2] cycloaddition upon exposure to wavelengths in the UV and revert back to the monomeric form upon exposure to wavelengths in the extremeUV, are also commonly employed for photoinduced shape change. When assembled into photomechanical crystals, they behave similarly to diarylethene and anthracene-based systems. For example, Kim et al. demonstrated that [2+2] dimerization of 4chlorocinammic acid drives twisting in sub- $\mu \mathrm{m}$ thick microribbons composed of oriented 1-D stacks [34] due to a mismatch strain between regions populated by dimers and monomers. Interestingly, while microribbons were shown to twist, rectangular prisms and plate-like crystals with the same crystal packing motifs but of $\mu \mathrm{m}$-scale thicknesses were not photoactive. The authors postulated that this is could be due to a difference in the density of defects between thinner and thicker crystals, incomplete photoexcitation in thicker crystals due to a greater ratio of thickness to penetration depth, decreased heat dissipation leading to melting in larger crystals, or decreased flexibility compared to thinner ribbons.

In addition to its use in photomechanical crystals, cinnamic acid is also widely used for dynamic crosslinking of shape-memory polymers to spatially program stress. After elastically stretching a film of material, dynamic cross-links can be photo-patterned to "lock-in" strains locally. When the material is released, the film buckles due to the mismatch in strain between crosslinked and uncrosslinked regions. As a demonstration of this strategy, Lendlein and co-workers synthesized networks containing cinnamic acid 
[35]. As shown in Fig. 3F, shapes were fixed by stretching the film, selectively crosslinking certain regions through a photomask, and allowing the rest of the film to relax to create 'temporary' shapes such as arches and spirals. Arches were programmed by exploiting non-uniform light absorption through the film such that the equilibrium length of the exposed side is greater than the opposite side due crosslinking, resulting in curling towards the shorter side. Spirals, however, were patterned by selectively crossing-linking stripes offset from the long axis of the film such that incompatible curvatures are introduced, similar to the mechanics of LCN ribbons presented earlier. Upon exposure to shorter wavelengths, the de-dimerization allowed the stressed regions to relax and the film to assume its original 'permanent' shape. Significantly, in this work, multiple temporary shapes can be maintained without the need for constant illumination through photoreversible dimerization.

\subsection{Bond Exchange}

Light-induced homolysis of covalent bonds into radicals is among the most common and useful classes of photochemical reactions. In the context of shape-programmable materials, a widely used method of this type is the addition-fragmentation chain transfer of allyl sulfides for photo-reversible cleavage and rearrangement of network chains within shape memory polymers [36]. In this method, a photoinitiator generates a propagating thiyl radical which can subsequently add to an allyl sulfide, producing an unstable intermediate that cleaves to generate a new thiyl radical. When polymer networks with incorporated allyl sulfides are placed under strain and illuminated, this bond exchange 
process relaxes stress as a result of rearrangements in network topology. Thus, instead of exploiting photo-crosslinking to lock-in stress as is the case with cycloadditions, shape is programmed in these systems by using photochemical reactions to relieve stress. Because light absorption, and thus radical formation, varies through the film thickness, a gradient of stress is induced that causes out-of-plane bending. In one particularly elegant example (shown in Fig. 1G), Ryu et al. used exploited this method in polymer sheets to create folded structures by selectively irradiating a uniaxially strained film to define fold regions [37]. The fold angle could be controlled by varying the width of the illuminated region with wider rectangular region giving tighter fold angles because of increased curvature. In this way, a closed 3-D cube was realized. This technique can also be extended to other materials systems. In a recent example, McBride et al. polymerized liquid crystal networks containing allyl sulfides for dynamic control over nematic ordering [38]. When an allyl sulfide is incorporated into an aligned LCN, exposure to spatially patterned light locally disrupts nematic ordering in the exposed region, allowing for spatially controlled programming of ordered and disordered regions. As shown in Figure $3 \mathrm{H}$, they exploited this effect to spatially control sample topography.

\section{Photothermal Materials}

Photothermal materials generate heat when illuminated with light. To generate shape change, photothermal heaters are typically combined with polymer matrices that undergo a dimensional change in response to a thermal transition. For example, driving expansion or contraction upon crossing the glass transition temperature $\left(T_{g}\right)$ in a polymer network, thermal de-swelling of hydrogels exhibiting lower critical solution temperature (LCST) 
behavior, or contraction along the director due to a reduction in order parameter of LCNs. In this section, we review light-driven shape programming based on three main types of photothermal heaters: 1) organic dyes, 2) carbon materials, and 3) metallic nanoparticles.

\subsection{Dyes}

In addition to their utility for transforming light into chemical changes, chrompophores can also drive shape change through the dissipation of heat into a matrix. Pioneering work using dyes as heat generators was conducted by Suzuki and Tanaka [39]. When chlorophyllin sidechains where incorporated into PNIPAAm gels, exposure to visible light produced heat that drove the gel to collapse. In the intervening thirty years, dyes have been incorporated into a variety of matrices to drive complex shape changes. 


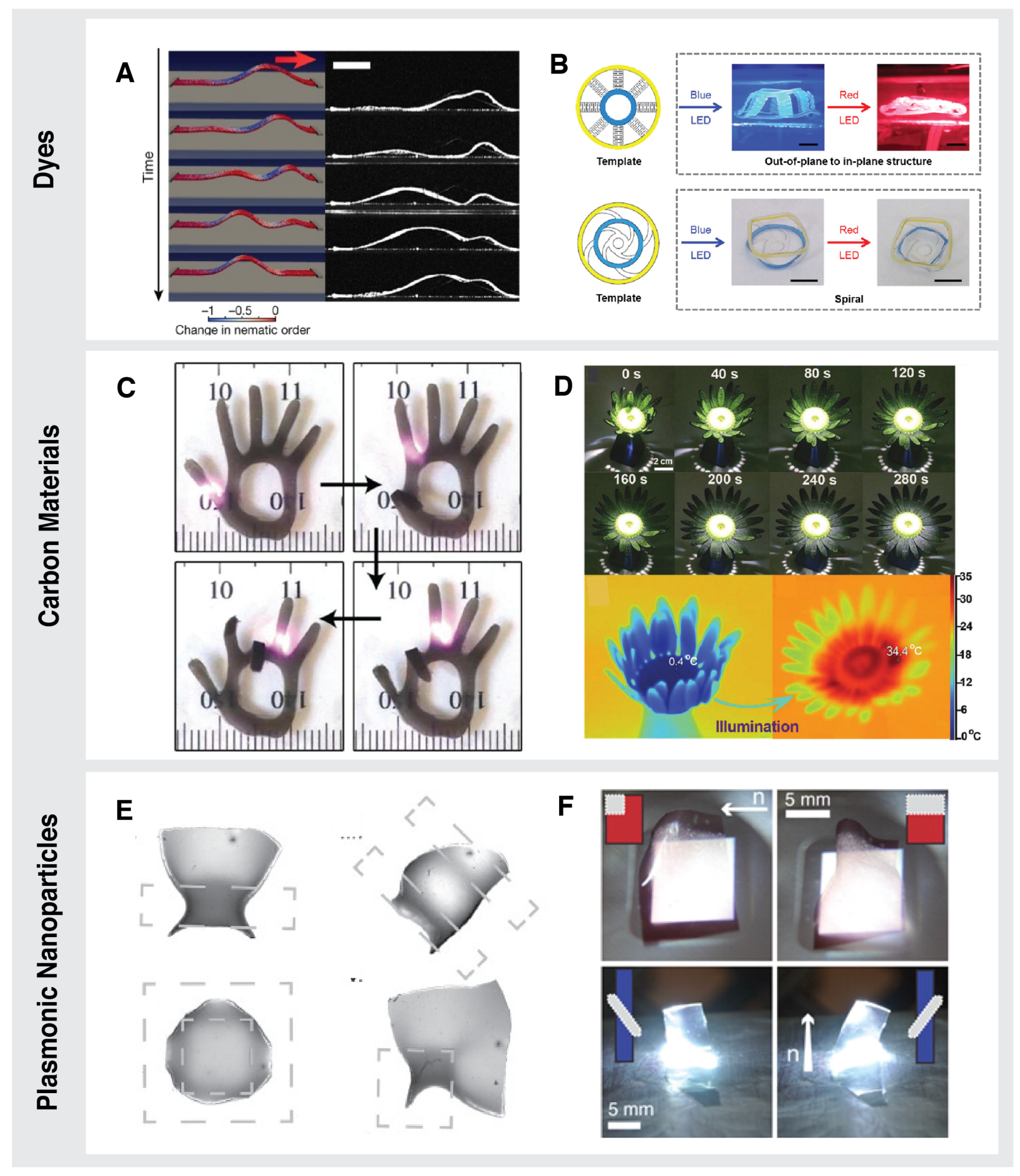

Figure 4. Examples of photothermal-driven shape change. (A) Wave formation in an LCN from heat generation via trans-cis-trans cycling of azobenzene (reproduced from ref. [41]; copyright 2017 Springer Nature). (B) Sequential bending of absorbing colored dyes in pre-stretched polystyrene (reproduced from ref. [43]; copyright 2017 AAAS). (C) Localized actuation of a graphene-functionalized gel (reproduced from ref. [44]; copyright 2013 American Chemical Society). (D) One-way shape memory of 3D printed polyurethane/carbon black composites (reproduced from ref. [45]; copyright 2017 Wiley). (E) Local deswelling of gold nanocomposite gel sheets (reproduced from ref. [26]; copyright 2015 Wiley). (F) Spatiallycontrolled buckling of glassy LCNs with incorporated gold nanoparticles (reproduced from ref. [48]; copyright 2016 American Chemical Society). 
Isomerizable dyes, often based on azobenzene, are among the most widely used photothermal dyes. So-called "push-pull" azobenzenes, where the phenyl rings are functionalized with opposite electron-withdrawing and electron-donating groups, generate heat by rapidly isomerizing between trans and cis isomers under blue-green light due to overlapping absorbance bands of the two isomers. In particular, two recent examples demonstrate the utility of this approach using DR1, an azobenzene-based dye, to drive photoinduced shape change in LCNs. Priimagi and co-workers spatially programmed the orientation of DR1-containing LCNs to fabricate multi-legged structures with splay director alignments, where rotation of the director from laying in to out of plane of the film through the film thickness drives the film to contract and expands orthogonally on opposite sides [40]. When exposed to light, the legs of these structures bend out-of-plane due to expansion and contraction along the same axis on opposite sides of the film, yielding light-responsive grippers and 'octopods'. In another recent example, Broer's group exploited the heat generation of DR1 to drive propagation of waves in LC films as shown in Fig. 3A [41]. By incorporating azo-dyes with increasingly short cis half-lives into a splay aligned film a transition from a stationary arc-like configuration to a continuous wave-like deformation when a film was illuminated from one side. Remarkably, due to the rapid cycling of the incoporated azo-compounds and self-shadowing effects in the film, stationary uniform illumination drives continuously regenerating motion and oscillatory shape change. Despite the utility of azobenzene in these contexts, prediction and characterization of the photoresponse are complicated by contributions to photomechanical work through both photochemical and photothermal mechanism, as 
alluded to in Section 3.1. However, recent work by Priimagi and co-workers using cooperative effects of azobenzene isomerization to program strain profiles and photoheating to deploy shape change demonstrates that the combination of photochemical and photothermal effects can be synergistically exploited under the right conditions [42].

Non-isomerizable visible and IR absorbers can also be used to drive actuation in non-LC films through purely photothermal means. For example, Dickey and co-workers used ink jet printers to deposit colored ink on the surface of pre-strained polystyrene sheets to absorb light in spatially defined regions to drive self-folding due to localized shrinkage [43]. Significantly, different colored inks produce heat in response to different wavelengths of light. When exposed to light of the correct wavelength, localized heat absorption in printed areas drives folding due to non-uniform heating - and thus incompatible strains through the film thickness. By locally inscribing hinges of different colors, origami and kirigami structures were folded sequentially according to the order of light exposure, as shown in Figure 4B. While folding is irreversible in this system, it allows for the programming of a wide variety of complex shapes with temporal control of structural evolution.

\subsection{Carbon Materials}

Due to their strong absorbance in the IR region, $\mathrm{sp}^{2}$-hybridized carbon materials such as carbon black, carbon nanotubes (CNT), and graphene are highly effective as photothermal materials. Polymer nanocomposites containing carbon-based materials 
have been demonstrated in a wide variety of systems to control shape, including thermoplastics, elastomers, LCNs, and gels. Here, we highlight a few recent and important examples of these systems.

As seen throughout this review, LCST hydrogels provide a useful platform for incorporating photoactive additives for photo-driven changes. For example, Wang et al. interfaced reduced graphene oxide (rGO) within porous thermoresponsive elastin-like peptides for near-IR responsive bending [44]. In their system, hydrogels were engineered with one side that was highly porous and one side that was relatively non-porous. The high porosity side swelled much faster than the low porosity side due to faster diffusion of water, leading to a through-thickness swelling gradient that drove bending towards the low-porosity side. When exposed to spatially-defined near-IR light, de-swelling of the gel caused localized bending to create a variety of curled shapes, leading to bending of fingers in an artificial hand (Figure 4C), directional curling of a gel disk, and motion of a light-driven crawler. More recently, Yang et al. used 3D printing of carbon black loaded polyurethane shape memory polymers to prepare 3D objects consisting of thin composite plates or struts [45]. The objects could be deformed into a new 3D shape at high temperature and cooled to set a distinct temporary shape. Upon exposure to IR light, the carbon black particles heat the object above the $T_{g}$ and return it to the initial printed shape. Remarkably, sunlight was sufficient to drive this transformation. As a proof-of-concept the authors demonstrated the printing, deformation, and recovery of cubic frames and "blooming" flowers, as shown in Fig. 4D. 


\subsection{Plasmonic Nanoparticles}

Among the most efficient and versatile photothermal heaters are metallic nanoparticles.

In particular, gold nanoparticles can have a photothermal conversion efficiency approaching unity for small nanoparticles and have a much greater absorption crosssections than dyes [46]. Heat generation in these materials occurs due to dissipation of heat from the collective oscillation of free electrons on the metal lattice surface-the surface plasmon resonance (SPR)-driven by resonant wavelengths of light. The wavelength at which maximum absorption occurs is determined by nanoparticle geometry and can be tuned through various synthetic methods. For example, the SPR of gold nanospheres can be tuned within the visible region by varying the wall thickness from solid spheres to thin-walled hollow nanoshells. Additionally, gold nanorods exhibit an additional SPR corresponding to the long axis of the particle that increases from the visible to the IR with increasing aspect ratio. While a variety of metals including gold, silver, cobalt, and palladium can be used, gold nanoparticles are perhaps the most widely studied due to their widely tunable SPRs and their ability to be functionalized with thiol ligands to promote colloidal stability.

The utility of gold nanoparticles in photothermal systems is illustrated by a number of examples in the literature. Sukhishvili et al. used layer-by-layer assembly of PNIPAAMgrafted gold nanospheres and nanoshells with non-responsive polymer to drive wavelength-selective deswelling in stratified structures [47]. Because of the different SPRs of these particles, distinct shapes were realized in response to specific 
wavelengths. For example, when a trilayer was fabricated with the outer layers containing IR-responsive nanoshells and the inner layer containing visible light-responsive nanospheres, a cross-like and hour-glass like shape were individually realized upon deployment with IR and visible light, respectively. Instead of using spatially-defined regions of wavelength-specific absorption to pattern multiple shape transitions in a single material, Hauser et al. used localized light exposure to deform PNIPAAm gels containing uniformly-dispersed gold nanospheres (Fig 4E) [26]. When exposed to spatially programmed patterns of white light, non-uniform strains caused by localized deswelling drove the formation of axisymmetric bottle-like shapes, helical rolls, domes, and wrinkled surfaces. Additionally, smooth swelling gradients could be achieved using grayscale patterns and directed motion was possible by sweeping stripes of light across the gel, as predicted by Balazs and co-workers [25]. Critically, this system is dynamically reconfigurable, as the gels rapidly reswell upon light removal. Expanding on this work, Hauser et al. incorporated gold nanoparticles into LCNs [48], and demonstrated buckling of films in hourglass shapes, helices, and cantilevers by combining patterns of light with different director orientations (Fig. 4F).

\section{Important Parameters Governing Light-Induced Shape Programming}

In this section, we discuss the principles governing the kinetics, efficiency, and spatial resolution of shape change in photochemical and photothermal materials. 


\subsection{Kinetics}

The photophysical processes underlying the responses of both photochemical and photothermal materials occur on very short time scales (e.g., a few ps or less). However, the observed timescale of deformation in photoactive materials is typically on the order of seconds or longer. Here we consider why these timescales are so different.

Photochemical transformations are ultimately limited by the rate of excited state relaxation, which is typically of order picoseconds for singlet-singlet transitions [49]. However, under typical light intensities used for addressing light responsive materials at least on the macroscopic scale, e.g., $I \sim 10^{4} \mathrm{~W} / \mathrm{m}^{2}$, the photon flux and small absorption cross-section of a typical photochrome mean that the characteristic time-scale for absorption of a photon is much longer, e.g., $\sim 1$ s. Further, the kinetics of shape transformation depend on the interplay between isomerization kinetics, light propagation, and mechanical properties of the matrix, often resulting in a lagged response. To understand these effects, we will consider isomerization of azobenzene, as it is among the most widely studied photochromes, though many of the general trends can be extended to other systems. In polymer solutions and in the rubbery state, illuminated samples will reach a photostationary state composed of both trans and cis isomers due to competing photoisomerization and thermal relaxation, the details of which are strongly influenced by molecular structure and matrix polarity, but only weakly dependent on viscosity [50]. However, in glassy matrices, trans-cis isomerization is generally slower due to the hindrance of molecular motion by the reduced free volume; the rate of reverse 
cis-trans thermal isomerization can also increase in the glassy state due to 'trapping' of the photogenerated cis isomers in a strained state [51]. Beyond single molecule isomerization, the shape evolution of an entire film is more complicated. For typical films were the thickness is much greater than the penetration depth, response times in both glassy and rubbery azobenzene systems can range from several seconds to several minutes due to progressive photobleaching through the film thickness $[19,52]$. Finally, while their role is not fully understood, viscoeleastic properties of the film also determine the response kinetics of the surrounding matrix. For example, while the timescale for cistrans thermal relaxation can be tuned from seconds to hours through molecular substitutions [53], a kinetic mismatch between azobenzene thermal relaxation and the observed mechanical response is often observed. Experiments by Broer [54,55] and White [19] suggest that crosslink density and $T_{g}$ influence the timescale of mechanical relaxation relative to chemical relaxation, though greater insight into the interplay of network relaxation and photoswitching kinetics is needed to fully understand and predict these phenomena.

For photothermal materials, the kinetics of macroscopic material response depends on heat transfer, which occurs on time scales that are highly dependent on the length-scales of interest, the material properties of the system, and the dominating heat transfer mechanism (i.e. conduction, convection, or radiation). We consider two simplified limits with relevance for shape-programming that are straightforward to understand. In the first case, we consider conduction-dominated transport for thin films embedded in an infinite 
medium of constant thermal conductivity, which is a reasonable approximation for hydrogel films in water with $\sim 10-100 \mu \mathrm{m}$ thicknesses and mm-scale lateral dimensions [26]. Illumination of a light-absorbing gel gives rise to a steady-state spatial variation in temperature within a time-scale of a few seconds, while the resulting change in shape is limited by the material response-in this case, the poroelastic swelling kinetics of the gel. In the second case, we consider heat transfer to the medium dominated by convection (or radiation), with negligible in-plane diffusion, a limit approached for very thin films with cm-scale lateral dimensions in air, as studied e.g. for LCN nanocomposites [48]. Here again, the time scale to reach thermal steady-state is typically a few seconds, while the viscoelastic material relaxation time may range from considerably faster to much slower.

\subsection{Efficiency}

The efficiency of work output from photomechanical systems, relative to either the incident or absorbed intensity of light, has not been well-characterized for many systems, but in general has so far been very low. This is due to a number of factors and is complicated by the interplay between optical and mechanical properties of photoactive materials. Here we examine several important factors governing work output in photochemical and photothermal systems and what is currently achievable.

The efficiency of the transduction of photochemical processes to mechanical work is complicated and is dependent on how the chromophore is incorporated into the matrix and the matrix properties itself. Single molecule AFM studies on azobenzenes 
incorporated into a polymer chain suggest that an energy conversion efficiency of $\sim 10 \%$ should be possible with a quantum yield of unity for trans to cis isomerization [56]. However, although quantum yields for photochemical reactions can approach unity in solution, these values generally decrease in solid matrices [51]. Beyond single molecule studies, where polymer chains are well-aligned into a configuration that should maximize force transduction, efficiencies in bulk materials are generally much lower due to the imperfect coordination between the responses of different photochromes. One consequence of low efficiencies is that the specific work output of photochemical actuators is also quite low. For example, while glassy azo-LCNs have elastic moduli on the order of GPa, and photo-induced stresses of up to 1 GPa have been predicted [57], photomechanical stresses that can typically be achieved are only several MPa. Additionally, matrix properties play a non-trivial role in determining the resulting work output: main-chain LC polymer generate more photo-work compared to side-chain LCs [58], higher $T_{g}$ materials generate greater stresses while lower $T_{g}$ materials transduce greater strains [59], and semi-crystallinity decreases work output [60].

Photothermal systems developed to date exhibit similarly limited efficiencies. The upper limit of efficiency that can be achieved by a system in converting heat to work is set by the Carnot efficiency $\eta=1-\frac{T_{C}}{T_{H}}$, where $T_{C}$ and $T_{H}$ are the temperatures of the cold and hot reservoirs, respectively. Within this constraint, a primary consideration is the temperature increase that can be achieved. While high intensity illumination can lead to temperature increases of many hundreds of degrees Kelvin in the local vicinity of 
individual chromophores or metallic nanoparticles [61], steady state temperature increases in bulk materials are generally far lower, typically $\sim 10-100 \mathrm{~K}$. As nanoparticle size increases, the efficiency of light conversion to heat can also decrease due to increased scattering [62]. Further, only a small amount of heat is actually converted into work, while the large majority is used to increase the internal energy of the matrix material, or lost to the surrounding environment, generally causing practical efficiencies to be far below the Carnot limit.

\subsection{Pattern Resolution}

While the spatial resolution of a pattern of light can ultimately reach the diffraction limit, the practical resolution with which deformation can be patterned may be substantially more limited. In the case of photochromes, if the photochemical transitions are assumed to be uncorrelated (i.e. transformation of one molecule has no effect on the transformation of neighboring molecules) then in principle, the boundaries between regions with differential photo-strains are limited by the optical resolution. In the case of photothermal materials, however, heat transfer will inevitably lead to some broadening. Again, we consider the two simple limits described in Section 5.1. For the conduction-dominated case, the steady-state temperature profile will be broadened compared to the pattern of illumination over a distance similar to the characteristic lateral dimensions of the heat producing regions. For the convection-dominated case, lateral broadening occurs over a length $(t k / h)^{1 / 2}$, where $h$ is the convective heat transfer coefficient and $k$ the thermal conductivity of the thin film. For a polymer film with $t \sim 100 \mu \mathrm{m}$ in air, this length-scale is 
of order $1 \mathrm{~mm}$, which is modest at least for patterning of $\mathrm{cm}$-scale films. However, even if the temperature field or distribution of photochemical transformations can be defined with very high resolution, elasticity of the film will limit the sharpness of deformations that can be achieved to those with radii of curvature at least several times the thickness of the film.

\section{Future Directions}

In this last section, we offer our perspective on the rational design of photomechanical materials and avenues for further development in the field.

\subsection{Design of Photoactive Elements}

The fundamental unit of any photoresponsive material is the element that transduces photon energy into a chemical or thermal response. Thus, the design of new photochemical or photothermal agents specifically engineered for the desired wavelength selectivity, efficiency, and reaction kinetics is necessary for the realization of novel photomechanical properties. While it is well-established that the optical properties and heat generation characteristics of nanoparticles are determined by their size and geometry, they are generally less easily tailored than organic chromophores. Organic chemistry provides a large toolbox for tailoring photochromes through chemical modifications such that variations are essentially limitless. For example, the effects of single substitutions on the spectroscopic characteristics and switching kinetics of azobenzene are well-studied. While unsubstituted azobenzene has an absorbance in the 
UV and a thermal relaxation time on the order of hours, both of these characteristics can be tailored through the addition of electron withdrawing or donating groups at the ortho and para positions. Halogenation at the ortho or para position results in absorption in the visible and thermal half-lives on the order of years [63] while addition of an electron donor and an acceptor group at the 4 and 4' positions, respectively, decreases the thermal relaxation time to the order of seconds [64]. While long relaxation times are desirable for shape persistence and all-optical control as recent work by Katsonis' group and White's group shows $[65,66]$, short relaxation times are useful for rapid switching and photoinduced motion $[41,67]$ and the ability to design photoswitches with responses tailored for the desired application is critical.

Beyond azobenzene, however, rational design of existing chromophores to demonstrate a desired photoresponse remains a challenge. In many cases, researchers rely on trialand-error approaches to empirically define the effects of molecular substitutions on photoswitching properties. However, empirical studies of large libraries of photoswitches is synthetically time-consuming and even thoughtful molecular design can often be little better than a shot in the dark. To circumvent these issues, computational tools can be used for the targeted design of photoactive compounds [68]. Thus, while synthesizing and characterizing multitudes of photoswitch derivatives is slow and cumbersome, quantum mechanical simulations of large libraries of chromophores can be used to inform targeted synthesis. 


\subsection{Solid State Engineering of Bulk and Composite Materials}

Photochemical or photothermal agents must be assembled into bulk materials or incorporated into matrices to translate molecular changes into macroscopic shape changes. However, how best to engineer the organization of such materials to generate the desired photoresponses is still an open question. For example, while photomechanical crystals can be grown into plates [29,34], microribbons [32], and nanorods [69] on the micron scale, it remains a challenge to grow crystals in other geometries and on larger length scales. Additionally, while efforts have been made to incorporate photomechanical crystals in to polymer matrices for larger energy densities without fracture $[70,71]$, it remains an engineering challenge to successfully generalize this approach to other systems.

While incorporation of photoactive materials into polymer films has a number of advantages including synthetic control of matrix properties, established processing protocols, and the ability to fabricate arbitrary geometries, there remain large gaps in the general understanding of how photoresponse is affected by the matrix properties. For example, azobenzene switching is known to be sensitive to viscosity, polarity, crystallinity, free volume, and segmental motion of the surrounding matrix [72]. Additionally, photosoftening in photoactive materials further complicates prediction and analysis of the resulting shape as the matrix properties dynamically change upon illumination [73]. In the case of gold or carbon nanomaterials, high loadings can stiffen the surrounding matrix and dampen the photoresponse. Understanding how all of these effects combine to 
generate a meaningful photoresponse is necessary for tailoring material actuation to specific applications.

\subsection{Patterning of Arbitrary Shapes}

Should all the challenges presented above be met, there would still remain the issue of how to rationally design arbitrary shape change in photomechanical materials. While the relatively simple case of designing materials with mean curvature via through-thickness strain gradients is well-understood, it is a much harder problem to design materials that deform into arbitrary 3D shapes with targeted distributions of mean and Gaussian curvature. As discussed in Section 2, light propagation and absorption through a photomechanical sheet couple with geometry and elasticity to further complicate this design problem. For example, light propagation and deformation are often coupled due to the effects of photobleaching, which has been shown to yield non-monotonic changes in shape with progressive illumination [74]. Thus, to fully capture the interplay of these effects, improved models capturing optical, photochemical, mechanical, and thermal effects are needed. In addition, optimization of these materials for practical applications require figures of merit to evaluate both the fidelity of realized shape change to the target design as well as the efficiency of light conversion to shape change and mechanical work.

\subsection{Light Delivery}

Finally, the ease with which light can be spatially, temporally, spectrally, and orientationally modulated presents possibilities for a high degree of dynamic and remote 

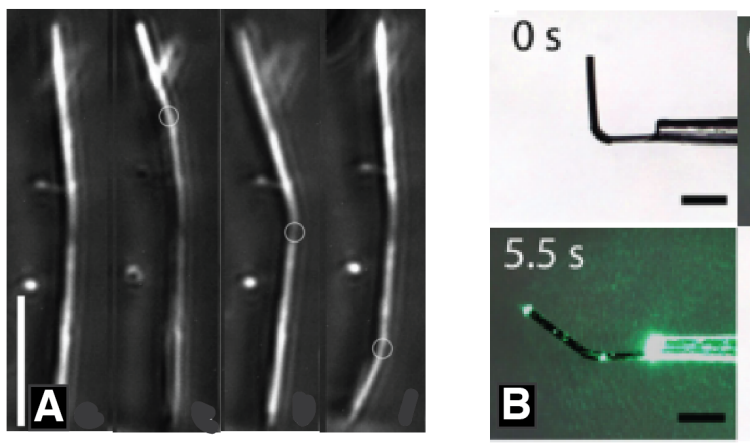

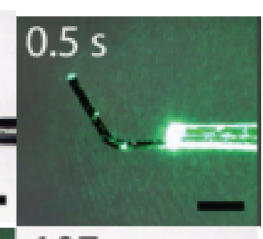

$107 \mathrm{~s}$

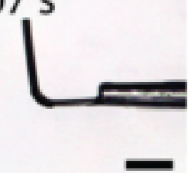

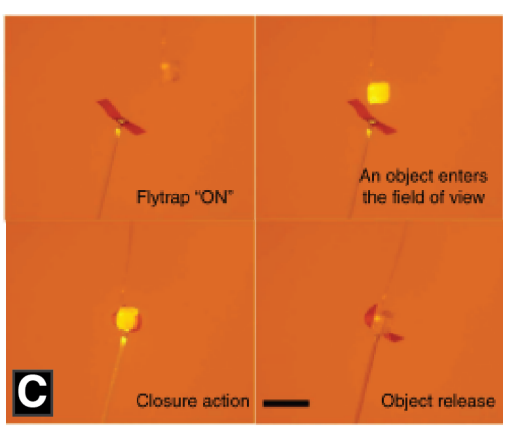

Figure 5. Photomechanical responses to different modes of light delivery. (A) Control of bending location in an anthracene-based photomechanical nanorod using 2PE (reproduced from ref. [75]; copyright 2009 Wiley). (B) Waveguiding actuation of a gel-photopolymer nanocomposite bilayer (reproduced from ref. [76]; copyright 2016 Wiley). (C) Actuation of a Venus fly trap-inspired LCN controlled by optical feedback (reproduced from ref. [77]; copyright 2017 Wani, Zeng, and Priimagi).

control of shape. As demonstrated in work by Hauser et al. and others [15,35], digital micromirror devices (DMDs) are a facile way to spatially control light delivery. However, higher resolution systems are needed to pattern light at smaller length scales for the miniaturization of photomechanical devices. One promising method for photoactuation on smaller length scales is through the use of two-photon excitation (2PE) to drive a highly localized response. For example, Bardeen and co-workers used 2PE to illuminate $\sim 1 \mu \mathrm{m}$ spots on anthracene nanorods of $35-200 \mathrm{~nm}$ in diameter (Figure $5 \mathrm{~A}$ ) to control the location, rate, and magnitude of bending [75]. Furthermore, 2PE can be used to control the depth of illumination in addition to the lateral dimensions of illumination, and this could potentially be exploited to drive rich, dynamic shape change in thick materials.

One drawback of using spatially patterned light to drive shape change is that it typically requires direct line-of-site illumination which, in some applications, is impractical or impossible. To circumnavigate these effects, waveguiding can be exploited to deliver light over long distances with a near-constant intensity or in confined spaces. For example, 
Zhou et al. exploited waveguiding to control optical fiber microactuators [76]. In this work, nanocomposite PNIPAAm gels were incorporated into bilayers with a photopolymer with excellent light transmission properties. When light was waveguided down the length of the fiber, photothermal deswelling of the nanocomposite gel drove bilayer bending (Figure 5B). To our knowledge, this method of light delivery has been demonstrated in very few photoactuation systems despite its advantages over flood illumination.

Finally, a major challenge in photomechanical systems is exploiting optical feedback for autonomous actuation. In these systems, modulation of illumination conditions due to external control or in response to shape change provides feedback to the systems to dynamically alter its response. In a recent example of this strategy, Wani et al. fabricated a Venus fly trap-inspired LCN actuator that spontaneously closed when a small object enters its field of view [77]. The splay-aligned LCN is initially flat and situated on top of an optical fiber. When a "fly" enters the field of view, the light is reflected back on the actuator and the small gripper closes around the object (Figure 5C). Once closed, the light is blocked and the gripper re-opens to release the object. Thus, harnessing optical feedback to trigger between multiple stable states is an important step towards autonomous devices and remains an open challenge in the field.

\section{Conclusions}

In this review, we have presented general strategies for shape control in synthetic systems, an overview of literature examples of photochemical and photothermal 
actuators, and offered our viewpoints on future work in this field. Photochemical actuators benefit from nearly limitless possible molecular structures, but their utility is limited by the properties of current generation photoswitches as well as limited ability to control their self-assembled structures. Photothermal actuators provide robust means for reversible actuation but require heat-responsive matrices and are generally limited to low efficiencies due to heat dissipation. Moving forward, new design rules are needed for the realization of arbitrary shapes driven by photoactuation and will require contributions from physics, chemistry, and engineering to realize the great potential of these materials.

\section{Conflict of Interest Statements}

Nothing declared.

\section{Acknowledgements}

This work was funded by the Office of Naval Research through the MURI on Photomechanical Material Systems (Award N00014-18-1-2624). The authors thank Profs. C. J. Bardeen, K. Bhattacharya, T. Martínez, P. Palffy-Muhoray, and J. Read de Alaniz for insightful discussions and suggestions.

\section{References}

Papers of particular interest, published within the period of review have been highlighted as:

* of special interest

* * of outstanding interest 
[1] Liu Y, Genzer J, Dickey MD. "2D or not 2D”: Shape-programming polymer sheets. Prog Polym Sci 2016;52:79-106. doi:10.1016/j.progpolymsci.2015.09.001.

[2] Zeng H, Wasylczyk P, Wiersma DS, Priimagi A. Light Robots: Bridging the Gap between Microrobotics and Photomechanics in Soft Materials. Adv Mater 2017;1703554:1703554. doi:10.1002/adma.201703554.

[3] Kim T, Zhu L, Al-Kaysi RO, Bardeen CJ. Organic photomechanical materials. ChemPhysChem 2014;15:400-14. doi:10.1002/cphc.201300906.

[4] Bisoyi HK, Urbas AM, Li Q. Soft Materials Driven by Photothermal Effect and Their Applications 2018;1800458:1-21. doi:10.1002/adom.201800458.

[5] White TJ, editor. Photomechanical Materials, Composites, and Systems: Wirelese Transduction of Light Into Work. 1st ed. Hoboken: John Wiley \& Sons, Ltd.; 2017.

[6] Stoney GG. The Tension of Metallic Films Deposited by Electrolysis. Proc R Soc A Math Phys Eng Sci 1909;82:172-5. doi:10.1098/rspa.1909.0021.

[7] Timoshenko BYS. Analysis of Bi-Metal Thermostats. J Opt Soc Am 1925;11:23355.

[8] Alben S, Balakrisnan B, Smela E. Edge effects determine the direction of bilayer bending. Nano Lett 2011;11:2280-5. doi:10.1021/nl200473p.

[9] Klein Y, Efrati E, Sharon E. Shaping of Elastic Sheets by Prescription of NonEuclidean Metrics. Science 2007;315:1116-20. doi:10.1126/science.1137920.

[10] Sydney Gladman A, Matsumoto EA, Nuzzo RG, Mahadevan L, Lewis JA. Biomimetic 4D printing. Nat Mater 2016;15:413-8. doi:10.1038/nmat4544.

[11] Aharoni H, Xia Y, Zhang X, Kamien RD, Yang S. Making Faces: Universal Inverse Design of Surfaces with Thin Nematic Elastomer Sheets. Proc Natl Acad Sci 2018;115:7206-11. doi:10.1073/pnas.1804702115.

[12] Plucinsky P, Kowalski BA, White TJ, Bhattacharya K. Patterning nonisometric origami in nematic elastomer sheets 2018:1-22.

[13] Aharoni H, Sharon E, Kupferman R. Geometry of Thin Nematic Elastomer Sheets. Phys Rev Lett 2014;113:257801. doi:10.1103/PhysRevLett.113.257801.

[14] Lee KM, White TJ. Photochemical Mechanism and Photothermal Considerations in the Mechanical Response of Monodomain, Azobenzene-Functionalized Liquid Crystal Polymer Networks. Macromolecules 2012;45:7163-70. 
doi:10.1021/ma301337e.

[15*] Finkelmann H, Nishikawa E, Pereira GG, Warner M. A new opto-mechanical effect in solids. Phys Rev Lett 2001;87:1-4. doi:10.1103/PhysRevLett.87.015501.

This paper is the first demonstration of photoisomerization in liquid crystal elastomers driving significant linear actuation.

$\left[16^{\star \star}\right]$ Yu Y, Nakano M, Ikeda T. Directed bending of a polymer film by light. Nature 2003;425:145-145. doi:10.1038/425145a.

One of the first demonstrations of reconfigurable shape change of a flat sheet via buckling driven by polarization-dependent isomerization of azobenzene in an LCN.

[17*] lamsaard S, Aßhoff SJ, Matt B, Kudernac T, Cornelissen JJLM, Fletcher SP, et al. Conversion of light into macroscopic helical motion. Nat Chem 2014;6:229-35. doi:10.1038/nchem.1859.

The authors determine design rules to control the shape selection of light-responsive helices using twisted nematic liquid crystal elastomers dope incorporating azobenzene and a chiral dopant.

[18] Aßhoff SJ, Lancia F, lamsaard S, Matt B, Kudernac T, Fletcher SP, et al. HighPower Actuation from Molecular Photoswitches in Enantiomerically Paired Soft Springs. Angew Chemie - Int Ed 2017;56:3261-5. doi:10.1002/anie.201611325.

[19** Ahn SK, Ware TH, Lee KM, Tondiglia VP, White TJ. Photoinduced Topographical Feature Development in Blueprinted Azobenzene-Functionalized Liquid Crystalline Elastomers. Adv Funct Mater 2016;26:5819-26. doi:10.1002/adfm.201601090.

This paper demonstrates complex shape changes using photoactuation of azobenzenedoped LCEs with patterned defects in director orientation.

[20*] Koshima H, Ojima N, Uchimoto H. Mechanical motion of azobenzene crystals upon photoirradiation. J Am Chem Soc 2009;131:6890-1. doi:10.1021/ja8098596.

This paper demonstrates the first examples of incorporating azobenzene into photomecahnical crystals.

[21] Bushuyev OS, Tomberg A, Friščić T, Barrett CJ. Shaping crystals with light: Crystal-to-crystal isomerization and photomechanical effect in fluorinated azobenzenes. J Am Chem Soc 2013;135:12556-9. doi:10.1021/ja4063019.

[22] Bushuyev OS, Singleton TA, Barrett CJ. Fast, reversible, and general photomechanical motion in single crystals of various Azo compounds using visible light. Adv Mater 2013;25:1796-800. doi:10.1002/adma.201204831.

[23] Bushuyev OS, Corkery TC, Barrett CJ, Friščić T. Photo-mechanical azobenzene 
cocrystals and in situ X-ray diffraction monitoring of their optically-induced crystalto-crystal isomerisation. Chem Sci 2014;5:3158-64. doi:10.1039/C4SC00987H.

[24] Klajn R. Spiropyran-based dynamic materials. Chem Soc Rev 2014;43:148-84. doi:10.1039/c3cs60181a.

[25*] Kuksenok O, Balazs AC. Modeling the photoinduced reconfiguration and directed motion of polymer gels. Adv Funct Mater 2013;23:4601-10. doi:10.1002/adfm.201203876.

While uniform illumination was historically used to photoactuation, this paper uses computational methods to show that dynamic, spatially-controlled light patterns can be used to drive complex shape change and motion in photoresponsive gels.

[26] Hauser AW, Evans AA, Na JH, Hayward RC. Photothermally reprogrammable buckling of nanocomposite gel sheets. Angew Chemie - Int Ed 2015;54:5434-7. doi:10.1002/anie.201412160.

[27] Stumpel JE, Ziółkowski B, Florea L, Diamond D, Broer DJ, Schenning APHJ. Photoswitchable ratchet surface topographies based on self-protonating spiropyran-NIPAAM hydrogels. ACS Appl Mater Interfaces 2014;6:7268-74. doi:10.1021/am500542f.

[28] Mamiya J, Kuriyama A, Yokota N, Yamada M. Photomobile Polymer Materials: Photoresponsive Behavior of Cross-Linked Liquid-Crystalline Polymers with Mesomorphic Diarylethenes. Chem - A Eur J 2015;21:3174-7. doi:10.1002/chem.201406299.

[29] Kobatake S, Takami S, Muto H, Ishikawa T, Irie M. Rapid and reversible shape changes of molecular crystals on photoirradiation. Nature 2007;446:778-81. doi:10.1038/nature05669.

[30*] Kitagawa D, Tsujioka H, Tong F, Dong X, Bardeen CJ, Kobatake S. Control of photomechanical crystal twisting by illumination direction. J Am Chem Soc 2018;140:4208-12. doi:10.1021/jacs.7b13605.

In this paper diarylethene ribbons are shown to evolve from helicoids to cylindrical helices depending on the angle of incident light; this is distinct from LCN ribbons where only one shape is observed during actuation.

[31*] Kim T, Zhu L, Mueller LJ, Bardeen CJ. Mechanism of photoinduced bending and twisting in crystalline microneedles and microribbons composed of 9methylanthracene. J Am Chem Soc 2014;136:6617-25. doi:10.1021/ja412216z.

The authors monitor strain as a function of dimerization in anthracene crystals in different geometries and develop a model that correlates reaction kinetics to strain generation. 
[32**] Zhu L, Al-Kaysi RO, Bardeen CJ. Reversible photoinduced twisting of molecular crystal microribbons. J Am Chem Soc 2011;133:12569-75.

doi:10.1021/ja201925p.

This paper is the first demonstration of photoinduced twisting under uniform illumination in photomecahnical crystals, the twist period of which is controlled by amount of light exposure and ribbon cross-section.

[33] Tong F, Xu W, Al-Haidar M, Kitagawa D, Al-Kaysi RO, Bardeen CJ. Photomechanically Induced Magnetic Field Response by Controlling Molecular Orientation in 9-Methylanthracene Microcrystals. Angew Chemie - Int Ed 2018;130:1-6. doi:10.1002/anie.201802423.

[34] Kim T, Zhu L, Mueller LJ, Bardeen CJ. Dependence of the solid-state photomechanical response of 4-chlorocinnamic acid on crystal shape and size. CrystEngComm 2012;14:7792. doi:10.1039/c2ce25811k.

[35**] Lendlein A, Jiang H, Yunger O, Langer R. Light-induced shape-memory polymers. Nature 2005;434:695-7. doi:10.1038/nature03438.1.

A seminal paper in the development of shape memory polymers, this paper demonstrates the power of reversible photocrosslinking to program arbitrary shapes in polymer sheets.

$\left[36^{\star *}\right]$ Scott TF, Schneider AD, Cook WD, Bowman CN. Photoinduced Plasticity in Cross-Linked Polymers. Science (80- ) 2005;308:1615-7. doi:10.1126/science.1110505.

This paper introduced the use of allyl sulfides for shape programming, the chemistry of which has been widely applied in a number of shape-changing systems.

[37*] Ryu J, D’Amato M, Cui X, Long KN, Jerry Qi H, Dunn ML. Photo-origami-Bending and folding polymers with light. Appl Phys Lett 2012;100. doi:10.1063/1.3700719.

This paper demonstrates a simple method for the creation of 3D objects from 2D sheets through the use of photo-induced stress relaxation to pattern through-plane stress gradients for folding of origami-inspired objects.

[38] McBride MK, Hendrikx M, Liu D, Worrell BT, Broer DJ, Bowman CN.

Photoinduced Plasticity in Cross-Linked Liquid Crystalline Networks. Adv Mater 2017;29:1606509. doi:10.1002/adma.201606509.

[39**] Suzuki A, Tanaka T. Phase transition in polymer gels induced by visible light. Nature 1990;346:345-7. doi:10.1038/346345a0.

This paper birthed the field of photoresponsive gels.

[40] Wani OM, Zeng $\mathrm{H}$, Wasylczyk $\mathrm{P}$, Priimagi A. Programming Photoresponse in Liquid Crystal Polymer Actuators with Laser Projector. Adv Opt Mater 2018;6:2-7. 
doi:10.1002/adom.201700949.

[41**] Gelebart AH, Jan Mulder D, Varga M, Konya A, Vantomme G, Meijer EW, et al. Making waves in a photoactive polymer film. Nature 2017;546:632-6.

doi:10.1038/nature22987.

Autonomous and self-propogating motion has long been an open challenge in self robotics and this paper demonstrates how photothermal dyes can be used in an LCN to drive repetitive motion.

[42] Lahikainen $\mathrm{M}$, Zeng $\mathrm{H}$, Priimagi A. Reconfigurable photoactuator through synergistic use of photochemical and photothermal effects. Nat Commun 2018:18. doi:10.1038/s41467-018-06647-7.

[43*] Liu Y, Shaw B, Dickey MD, Genzer J. Sequential self-folding of polymer sheets. Sci Adv 2017;3:1-8. doi:10.1126/sciadv.1602417.

While self-folding has been demonstrated in many systems, this paper introduces a facile way to spatiotemporally control multi-step shape change using commercially available materials.

[44] Wang E, Desai MS, Lee SW. Light-controlled graphene-elastin composite hydrogel actuators. Nano Lett 2013;13:2826-30. doi:10.1021/nl401088b.

[45] Yang H, Leow WR, Wang T, Wang J, Yu J, He K, et al. 3D Printed Photoresponsive Devices Based on Shape Memory Composites. Adv Mater 2017;29:1-7. doi:10.1002/adma.201701627.

[46] Qin Z, Wang Y, Randrianalisoa J, Raeesi V, Chan WCW, Lipiński W, et al. Quantitative Comparison of Photothermal Heat Generation between Gold Nanospheres and Nanorods. Nat Publ Gr 2016:1-13. doi:10.1038/srep29836.

[47] Zhu Z, Senses E, Akcora P, Sukhishvili SA. Programmable light-controlled shape changes in layered polymer nanocomposites. ACS Nano 2012;6:3152-62. doi:10.1021/nn204938j.

[48*] Hauser AW, Liu D, Bryson KC, Hayward RC, Broer DJ. Reconfiguring Nanocomposite Liquid Crystal Polymer Films with Visible Light. Macromolecules 2016:acs.macromol.6b00165. doi:10.1021/acs.macromol.6b00165.

This work combines director patterning and spationtemporal light control in LCNs dope with gold nanoparticles to reversibly reconfigure $2 \mathrm{D}$ sheets into a variety of $3 \mathrm{D}$ shapes.

[49] Bandara HMD, Burdette SC. Photoisomerization in different classes of azobenzene. Chem Soc Rev 2012;41:1809-25. doi:10.1039/c1cs15179g.

[50] Serra F, Terentjev EM. Effects of solvent viscosity and polarity on the 
isomerization of azobenzene. Macromolecules 2008;41:981-6.

doi:10.1021/ma702033e.

[51] Paik CS, Morawetz H. Photochemical and Thermal Isomerization of Azoaromatic Residues in the Side Chains and the Backbone of Polymers in Bulk1,2. Macromolecules 1972;5:171-7. doi:10.1021/ma60026a015.

[52] Van Oosten CL, Corbett D, Davies D, Warner M, Bastiaansen CWM, Broer DJ. Bending dynamics and directionality reversal in liquid crystal network photoactuators. Macromolecules 2008;41:8592-6. doi:10.1021/ma801802d.

[53] Yager KG, Barrett CJ. All-optical patterning of azo polymer films. Curr Opin Solid State Mater Sci 2001;5:487-94. doi:10.1016/S1359-0286(02)00020-7.

[54] Liu D, Broer DJ. New insights into photoactivated volume generation boost surface morphing in liquid crystal coatings. Nat Commun 2015;6:1-7. doi:10.1038/ncomms9334.

[55] Hendrikx M, Schiphorst J, Heeswijk EPA Van, Koçer G, Knie C, Bléger D, et al. $\mathrm{Re}$ - and Preconfigurable Multistable Visible Light Responsive Surface Topographies. Small 2018;14:1803274. doi:10.1002/smll.201803274.

[56**] Hugel T, Holland NB, Cattani A, Moroder L, Seitz M, Gaub HE. Single-molecule optomechanical cycle. Science (80- ) 2002;296:1103-6.

doi:10.1126/science.1069856.

This paper measures the force generation from a single azobenzene-containing polymer chain and gives an estimate of the available specific work and efficiency.

[57] Toshchevikov V, Ilnytskyi J, Saphiannikova M. Photoisomerization Kinetics and Mechanical Stress in Azobenzene-Containing Materials. J Phys Chem Lett 2017;8:1094-8. doi:10.1021/acs.jpclett.7b00173.

[58] Sánchez-Ferrer A, Merekalov A, Finkelmann H. Opto-Mechanical Effect in Photoactive Nematic Side-Chain Liquid-Crystalline Elastomers. Macromol Rapid Commun 2011;32:671-8. doi:10.1002/marc.201100005.

[59] Wermter $\mathrm{H}$, Finkelmann $\mathrm{H}$. Liquid crystalline elastomers as artificial muscles. EPolymers 2001;1:111-23. doi:10.1515/epoly.2001.1.1.111.

[60] Lee KM, Wang DH, Koerner H, Vaia RA, Tan L-S, White TJ. Enhancement of Photogenerated Mechanical Force in Azobenzene- Functionalized Polyimides. Angew Chemie 2012;124:4193-7. doi:10.1002/ange.201200726.

[61] Fang GJ, MacLennan JE, Yi Y, Glaser MA, Farrow M, Korblova E, et al. Athermal 
photofluidization of glasses. Nat Commun 2013;4. doi:10.1038/ncomms2483.

[62] Govorov AO, Richardson HH. Generating heat with metal nanoparticles. Nano Today 2007;2:30-8. doi:10.1016/S1748-0132(07)70017-8.

[63] Bléger D, Schwarz J, Brouwer AM, Hecht S. O -fluoroazobenzenes as readily synthesized photoswitches offering nearly quantitative two-way isomerization with visible light. J Am Chem Soc 2012;134:20597-600. doi:10.1021/ja310323y.

[64] Mahimwalla Z, Yager KG, Mamiya JI, Shishido A, Priimagi A, Barrett CJ. Azobenzene photomechanics: Prospects and potential applications. vol. 69. 2012. doi:10.1007/s00289-012-0792-0.

[65*] lamsaard S, Anger E, Aßhoff SJ, Depauw A, Fletcher SP, Katsonis N. Fluorinated Azobenzenes for Shape-Persistent Liquid Crystal Polymer Networks. Angew Chemie Int Ed 2016:1-6. doi:10.1002/anie.201603579.

This paper demonstrates how changing the thermal relaxation time of azobenzene can be exploited for shape fixity in LCNs.

[66] Donovan BR, Matavulj VM, Ahn S kyun, Guin T, White TJ. All-Optical Control of Shape. Adv Mater 2018;31:1-7. doi:10.1002/adma.201805750.

[67] Gelebart AH, Vantomme G, Meijer EW, Broer DJ. Mastering the Photothermal Effect in Liquid Crystal Networks: A General Approach for Self-Sustained Mechanical Oscillators 2017. doi:10.1002/adma.201606712.

[68] Oruganti B, Wang J, Durbeej B. Quantum chemical design of rotary molecular motors. Int J Quantum Chem 2018;118:1-16. doi:10.1002/qua.25405.

[69] Al-Kaysi RO, Bardeen CJ. General method for the synthesis of crystalline organic nanorods using porous alumina templates. Chem Commun 2006:1224-6. doi:10.1039/b516732a.

[70] Lan T, Chen W. Angewandte Hybrid Nanoscale Organic Molecular Crystals Assembly as a Photon- Controlled Actuator 2013:6496-500. doi:10.1002/anie.201300856.

[71] Koshima H, Matsudomi M, Uemura Y, Kimura F, Kimura T. Light-driven Bending of Polymer Films in Which Salicylidenephenylethylamine Crystals are Aligned Magnetically. Chem Lett 2013;42:1517-9. doi:10.1246/cl.130797.

[72] White TJ. Light to work transduction and shape memory in glassy, photoresponsive macromolecular systems: Trends and opportunities. J Polym Sci Part B-Polymer Phys 2012;50:877-80. doi:10.1002/polb.23079. 
[73] Kumar J, Li L, Jiang XL, Kim DY, Lee TS, Tripathy S. Gradient force: The mechanism for surface relief grating formation in azobenzene functionalized polymers. Appl Phys Lett 1998;72:2096-8. doi:10.1063/1.121287.

[74*] Corbett D, Xuan C, Warner M. Deep optical penetration dynamics in photobending. Phys Rev E - Stat Nonlinear, Soft Matter Phys 2015;92:8-12. doi:10.1103/PhysRevE.92.013206.

Presents a model to predict the photostationary and dynamic states of deformation in LCNs as a function of the incident angle of illumination and accounts for self-shadowing effects.

[75] Good JT, Burdett JJ, Bardeen CJ. Using two-photon excitation to control bending motions in molecular-crystal nanorods. Small 2009;5:2902-9. doi:10.1002/smll.200900895.

[76] Zhou Y, Hauser AW, Bende NP, Kuzyk MG, Hayward RC. Waveguiding Microactuators Based on a Photothermally Responsive Nanocomposite Hydrogel. Adv Funct Mater 2016;26:5447-52. doi:10.1002/adfm.201601569.

[77] Wani OM, Zeng $\mathrm{H}$, Priimagi A. A light-driven artificial flytrap. Nat Commun 2017;8:15546. doi:10.1038/ncomms15546. 


\section{Graphical Abstract}

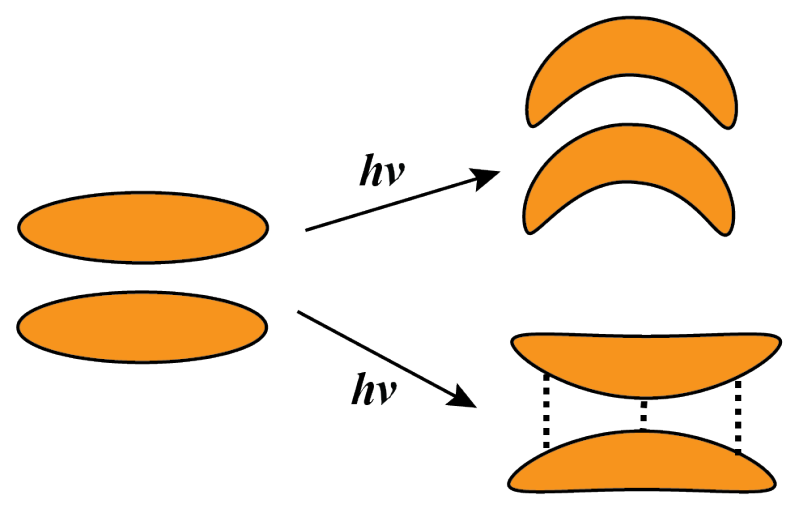

Photochemical molecules

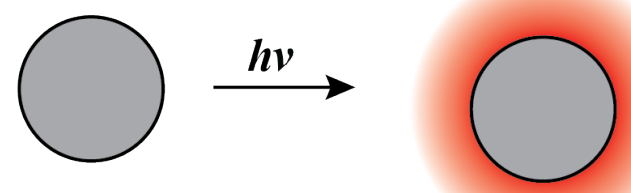

Photothermal heaters

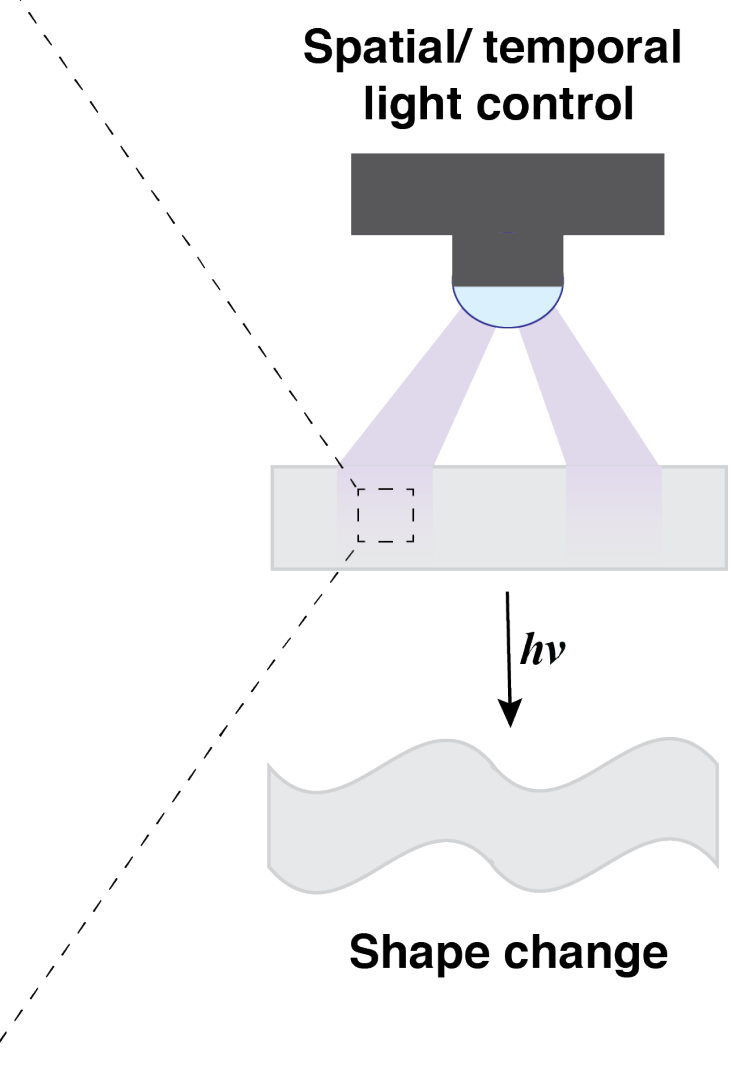

